\title{
Differential cytology of bronchoalveolar lavage fluid in immunosuppressed children with pulmonary infiltrates
}

\author{
F Ratjen, U Costabel, W Havers
}

\begin{abstract}
Objective-Bronchoalveolar lavage (BAL) is a well established technique for the detection of pathogens in immunosuppressed children, but its diagnostic yield is variable. The aim of this study was to investigate whether BAL differential cell counts are helpful in the evaluation of pulmonary infiltrates in immunocompromised children.
\end{abstract}

Study design-BAL was performed 28 times in 27 febrile immunocompromised children with pulmonary infiltrates. All patients were pretreated with broad spectrum antibiotics; 11 children also received amphotericin B. BAL was conducted with a flexible bronchoscope wedged in the area of maximal pathology as suggested by the chest radiograph or in the middle lobe in patients with diffuse interstitial radiographic changes. Differential cell counts were performed from cell smears obtained after centrifugation of BAL fluid. Results-Bacterial or fungal organisms were detected in BAL fluid of 12 patients. Patients with bacterial or fungal infections (group 1) had a significantly higher percentage of granulocytes in BAL fluid both compared with patients with sterile BAL cultures (group 2) and with a control group of children without pulmonary disease ( $p<0.001$, Wilcoxon test). The proportion of lymphocytes was not different from the control group in group 1 but significantly increased in group $2(p<0.001$, Wilcoxon test). Blood differential cell counts were not different in the two patient groups. Lymphocyte subsets of BAL fluid obtained in a subgroup of patients were not significantly different from controls.

Conclusion-These data suggest that BAL differential cell counts may be a useful adjunct in the differential diagnosis of pulmonary infection in immunocompromised children.

(Arch Dis Child 1996;74:507-511)

Keywords: bronchoalveolar lavage, immunosuppression, pulmonary infiltrates.

Pulmonary infiltrates in immunosuppressed patients are a challenge to the physician as both clinical signs and the radiological appearance are non-specific and can be caused by a wide spectrum of pathogens. ${ }^{1}$ In recent years, bronchoalveolar lavage (BAL) has emerged as a sensitive technique for achieving a specific
Table 1 Clinical diagnosis of the study population

\begin{tabular}{ll}
\hline Diagnosis & No \\
\hline After BMT due to leukaemia & 8 \\
Acute myeloid leukaemia & 8 \\
Acute lymphoblastic leukaemia & 5 \\
AIDS & 2 \\
Severe combined immunodeficiency & 1 \\
Aplastic anaemia & 1 \\
Medulloblastoma & 1 \\
Rhabdomyosarcoma & 1 \\
\hline
\end{tabular}

BMT = bone marrow transplantation.

diagnosis in these children. ${ }^{2-10}$ As the technique is moderately invasive and requires deep sedation or anaesthesia, at least in younger children, it is generally reserved for cases where empiric treatment fails. This implies that children undergoing BAL are likely to be treated with broad spectrum antibiotics, which lessens the likelihood that the underlying organism is detected with the procedure. In adults, the assessment of the local immune response as reflected in BAL fluid cytology has been found to be useful in the diagnosis of infectious pulmonary infiltrates. ${ }^{11}$ Studies in children have been hampered by the lack of reference values for this age group. We have recently reported differential cell counts of BAL fluid in children without pulmonary disease. ${ }^{12}$ To investigate whether the assessment of differential cytology is helpful in the differential diagnosis of pneumonia in immunocompromised paediatric patients, we have studied BAL cells in immunosuppressed children with pulmonary infiltrates.

\section{Patients and methods}

Twenty seven immunosuppressed children undergoing BAL between June 1992 and June 1995 in our centre were studied. Their mean (SD) age was 8.2 (5.3) years, range 1-19 years. Table 1 describes their diagnoses. BAL results of the immunosuppressed children were compared with a control group of children undergoing BAL during elective surgery for non-pulmonary illnesses. The characteristics of this population have been described in detail before. ${ }^{13}{ }^{14}$ The study was approved by the ethics committee of our institution; informed consent from both parents was obtained for all cases.

Immunosuppressed children received cotrimoxazole for prophylaxis of Pneumocystis carinii infection before the onset of their pulmonary illness. Chest radiograph changes varied from local infiltrates to diffuse interstitial changes. All patients were febrile and received broad spectrum antibiotics at the time of BAL. Eleven children were also treated with amphotericin B. One child underwent BAL 
twice; the second BAL in this patient was combined with a transbronchial biopsy, which was not performed in any other subject. Total of 28 samples of BAL fluid were thus available for analysis.

BAL was performed under general anaesthesia in all but the oldest subject who was studied under local anaesthesia with lidocaine. Children received flunitrazepam $(0.04-0.05 \mathrm{mg} / \mathrm{kg}$ body weight) orally one hour before the procedure. In children under 6 years anaesthesia was initiated by inhalation of a nitrous oxide-oxygen mixture with 2.5 volume (vol) \% halothane. Subsequently venous access was achieved and atropine (0.01 $\mathrm{mg} / \mathrm{kg}$ body weight), vecuronium $(0.05 \mathrm{mg} / \mathrm{kg}$ body weight), and alfentanil $(0.05 \mathrm{mg} / \mathrm{kg}$ body weight) were given intravenously. The halothane concentration was then reduced to $1.5 \mathrm{vol} \%$. In children older than 6 years venous access was obtained first and methohexitone $1-1.5 \mathrm{mg} / \mathrm{kg}$ body weight was given followed by atropine, vecuronium, and alfentanil as described above. After intubation pure oxygen was given with 1.5 vol \% halothane for the bronchoscopy.

A flexible bronchoscope (Pentax 3.5 or 4.9 $\mathrm{mm}$ ) was introduced through an adapter attached to the endotracheal tube thereby maintaining mechanical ventilation. The bronchoscope was wedged in the area of maximal pathology as suggested by the radiograph or in the middle lobe in patients with diffuse interstitial changes. BAL was performed with normal saline warmed to body temperature $\left(37^{\circ} \mathrm{C}\right) ; 1 \mathrm{ml} / \mathrm{kg}$ body weight was instilled and immediately withdrawn three times.

In subjects weighing less than $20 \mathrm{~kg}, \mathrm{BAL}$ was performed in three equal portions. In children weighing more than $20 \mathrm{~kg}, 20 \mathrm{ml}$ portions were instilled up to a total volume of $3 \mathrm{ml} / \mathrm{kg}$ body weight. The first fluid sample was used for bacterial and fungal studies only; subsequent samples were pooled for analysis. Both samples were stained by the Gram, ZiehlNeelsen, and potassium hydroxide methods. In addition, bacterial, fungal, and viral cultures as well as silver stains for $P$ carinii were performed on the pooled BAL fluid samples.

For the analysis of BAL cells, BAL fluid was filtered through sterile gauze and centrifuged at $500 \mathrm{~g}$ for 10 minutes. The cell suspension was washed three times in Eagle's minimal essential medium (MEM) containing $0.2 \%$ bovine serum albumin and $0.1 \%$ EDTA and resuspended in MEM. Bronchoalveolar cells were counted and cell viability was assessed by the trypan blue exclusion test. Differential cell counts were obtained from smears stained with May-Grünwald-Giemsa. At least 600 cells were counted in each subject.

Lymphocyte surface markers were assessed with the peroxidase antiperoxidase method. This technique has been described in detail elsewhere. ${ }^{15}$ In summary $10 \mu \mathrm{l}$ of the cell suspension are incubated on commercially available adhesive glass slides (Bio-Rad). Attached cells are fixed with a $0.05 \%$ solution of glutaraldehyde to block Fc receptors and preserve cell morphology. After washing with $0.3 \mathrm{M}$ glycine buffer to prevent binding of monoclonal antibodies to free aldehyde groups, preincubation with a
Table 2 Pathogens isolated from $B A L$ fluid

\begin{tabular}{ll}
\hline Pathogen & No \\
\hline Aspergillus fumigatus & 6 \\
Pseudomonas aeruginosa & 2 \\
Serratia marescens & 1 \\
Staphylococcus aureus & 1 \\
Pneumococcus & 1 \\
\hline
\end{tabular}

gelatin medium containing MEM with $0.2 \%$ gelatin Merck and $0.1 \%$ bovine serum albumin buffered with HEPES (pH 7.4) was undertaken for at least 15 minutes to reduce non-specific binding of immunoglobulins to glass and cells. The slides were incubated with monoclonal antibodies to surface antigens for 15 minutes. The following antibodies were used: OKT3 (CD3), OKT4 (CD4), and OKT8 (CD8) purchased from Ortho Diagnostic Systems, $\mathrm{B}_{1}$ (CD20), $\mathrm{IL}_{2} \mathrm{R}_{1}$ (CD25) from Coulter Electronics, and Leu 7 (CD57) as well as Leu 19 (HLA-DR) from Becton Dickinson. The incubation with monoclonal antibodies was followed by rabbit antimouse immunoglobulin and swine antirabbit immunoglobulin for 10 minutes each. Rabbit peroxidaseantiperoxidase complex was added for $10 \mathrm{~min}$ utes. Peroxidase activity was assessed by staining with diaminobenzidine-hydrogen peroxide followed by postfixation with osmium tetraoxide. The slides were covered with glycine and a cover glass. Positive staining cells exhibit dark brown granular staining on the cell membrane visualised under a light microscope with a 400-1000-fold magnification. At least 200 cells of each reaction were counted.

Leucocyte concentrations of peripheral blood were obtained on the day of BAL using an automatic cell counter (Coulter). Differential cell counts of peripheral blood were performed on smears stained with MayGiemsa-Grünwald. At least 100 leucocytes were counted in every patient. C reactive protein as a marker of inflammation was determined by immunonephelometry (Beckmann).

\section{STATISTICAL ANALYSIS}

All data were tested for normal distribution with the Kolmogorov-Smirnov test. Cell counts were expressed both as cells $/ \mathrm{ml}$ BAL fluid recovered and as a percentage of total cells. Results were expressed as mean (SD). The median is also reported for all data. Linear regression analysis was performed to assess the age dependency of the variables. Differences between the cellular composition of BAL fluid between normal children and immunosuppressed patients with and without bacterial or fungal agents in the fluid were assessed with the Wilcoxon-Mann-Whitney $\mathrm{U}$ test. A p value of less than 0.05 was considered as statistically significant.

\section{Results}

BAL was well tolerated in all patients. No complications occurred that could be related to the procedure except for an increased cough in the first hours after BAL that subsided after administration of a single dose of codeine. Infectious organisms were detected in the BAL fluid of 11 patients (table 2). In one patient BAL was sterile but Paecilomyces variotii, a fun- 
Table 3 Absolute cell counts of BAL fuid; values are mean (SD)

\begin{tabular}{lllllll}
\hline $\begin{array}{l}\text { Absolute cell counts } \\
\text { (cells/ml BAL fluid) }\end{array}$ & $\begin{array}{l}\text { Controls* } \\
(n=48)\end{array}$ & $\begin{array}{l}\text { Group 1 } \\
(n=12)\end{array}$ & pValuet & $\begin{array}{l}\text { Group } 2 \\
(n=16)\end{array}$ & pValuet & $p$ Value \\
\hline Total cells $\left(\times 10^{3}\right)$ & $10.3(11.1)$ & $48.3(56.5)$ & 0.02 & $22.4(27.5)$ & 0.049 & 0.26 \\
Macrophages & $8.3(8.6)$ & $13.0(11.3)$ & 0.30 & $6.7(4.4)$ & 0.61 & 0.30 \\
Lymphocytes & $1.8(3.4)$ & $4.3(3.2)$ & 0.01 & $14.5(25.3)$ & 0.008 & 0.62 \\
Neutrophils & $0.3(0.4)$ & $31.4(48.8)$ & 0.0002 & $0.8(1.2)$ & 0.77 & 0.003 \\
\hline
\end{tabular}

* From Ratjen et al. ${ }^{12}$

Group 1: immunocompromised patients with bacterial or fungal infection; group 2: immunocompromised patients without organisms in BAL fluid.

† Wilcoxon test, compared with controls. $¥$ Wilcoxon test, group $1 v$ group 2 .

gus with similarities to aspergillus, was cultured from a transbronchial biopsy specimen. Viral cultures were negative in all patients.

Total cell counts were higher in patients with pulmonary infiltrates when compared with children without bronchopulmonary disease (table 3). ${ }^{12}$ Differential cell counts of patients in whom fungal or bacterial organisms were detected on BAL had a significantly higher percentage of neutrophils than the control group of normal children (fig 1$)(p<0.001)$. A similar relationship was found for total neutrophil cell counts of BAL fluid (table 3). BAL neutrophil cell counts were also significantly higher compared with the group of children in which no bacterial or fungal agent was detected (table 3 ). The percentage of lymphocytes in children with bacteria or fungi in the BAL fluid was not different from the control group whereas absolute lymphocyte counts tended to be higher (fig 1, table 3).

In contrast, a significantly increased percentage of BAL lymphocytes as well as higher absolute lymphocyte counts were observed in

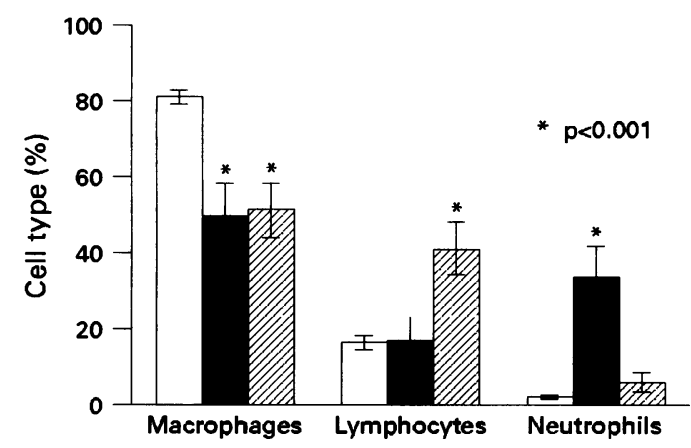

Figure 1 Differential cell counts of BAL fluid in per cent of total cells of immunosuppressed children with bacterial or fungal infection (open bars) and immunsuppressed children with negative BAL fluid cultures (hatched bars) compared with children without bronchopulmonary disease (closed bars). Data are expressed as mean (SEM). The percentage of macrophages is decreased in both patient groups $(p<0.001$, Wilcoxon test). Neutrophils are significantly increased in children with bacterial or fungal infections whereas a higher percentage of lymphocytes was observed in those with negative cultures ( $p<0.001$, Wilcoxon test). immunosuppressed patients without fungal or bacterial infectious organisms in BAL fluid (fig 1, table 3). Eosinophilic neutrophils as well as mast cells were within the normal range in both patient groups.

When the normal range was defined as cell counts within 2 SD of the control group, ${ }^{12}$ an increase in the proportion of neutrophils could be observed in 13 patients. In 10 of these 13 patients a bacterial or fungal organism was detected in BAL fluid. Of the patients with positive bacterial or fungal cultures only two had a normal percentage of neutrophils in BAL fluid. The first patient had aspergillus infection and absolutely no neutrophils in either peripheral blood or BAL fluid. The second patient was the one individual whose $\mathrm{BAL}$ was negative but in whom paecilomyces was found on transbronchial biopsy. This patient was the only subject with positive BAL cultures who showed an increased proportion of BAL lymphocytes. All other patients, in whom we found bacteria or fungi in BAL fluid, had lymphocyte cell counts within the normal range.

Differential cell counts of peripheral blood were not significantly different between the two patient groups, although neutrophil cell counts tended to be higher in patients in whom bacteria or fungi were detected in BAL fluid (table 4). C reactive protein, an acute phase reactant which is used as a marker of inflammation, was raised in both patient groups

Lymphocyte subsets of BAL fluid obtained in a subgroup of patients were highly variable and not significantly different to those of children without bronchopulmonary disease for most parameters (table 5). ${ }^{13} \mathrm{CD} 4$ cells tended to be increased in children with negative BAL fluid cultures resulting in a higher CD4/CD8 ratio in this group. HLA-DR expression as a non-specific sign of cell activation was increased in both patient groups.

\section{Discussion}

Pulmonary infections are one of the most frequent complications in immunosuppressed

Table 4 Blood differential cell counts in immunocompromised children with bacterial or fungal infection (group 1) compared with immunocompromised children with negative BAL cultures (group 2); values are mean (SD)

\begin{tabular}{llll}
\hline & Group 1 & Group 2 & p Value (Wilcoxon test) \\
\hline Leucocytes $\times 10^{9} / 1$ & $6.3(5.4)$ & $2.9(3.4)$ & 0.09 \\
Range & $0.1-17.5$ & $01-12.4$ & 0.09 \\
Neutrophils & $4.1(3.8)$ & $1.4(2.3)$ & \\
Range & $0-10$ & $0-8.6$ & 0.61 \\
Lymphocytes & $1.3(1.3)$ & $0.9(0.8)$ & \\
Range & $0.1-4.4$ & $0.1-3$ & 0.77 \\
CRP $(\mathrm{mg} / \mathrm{l})$ & $114(96)$ & $0-490$ & \\
Range & $0-290$ & $0.155)$ & \\
\hline
\end{tabular}

$\mathrm{CRP}=\mathrm{C}$ reactive protein. 
Table 5 Lymphocyte surface markers of BAL cells; values are mean(SD)

\begin{tabular}{llccc}
\hline Cell type (\% of lymphocytes) & Controls $^{*}(n=28)$ & Group 1 $(n=4)$ & Group 2 ( $n=9)$ & $p$ Valuet \\
\hline CD3 (T cells) & $86.0(5)$ & $86.0(6)$ & $84.0(9)$ & 0.76 \\
CD4 & $33.0(13)$ & $26.0(26)$ & $49.0(23)$ & 0.05 \\
CD6 & $0.8(1.8)$ & $0.3(0.3)$ & $0.3(0.3)$ & 0.21 \\
CD8 & $57.0(13)$ & $62.0(27)$ & $43.0(21)$ & 0.04 \\
CD4/CD8 & $0.7(0.4)$ & $0.7(0.8)$ & $2.0(2.4)$ & 0.07 \\
CD20 (B cells) & $0.9(1.4)$ & $0.4(0.8)$ & $0.7(0.6)$ & 0.60 \\
CD57 (NK cells) & $7.8(8.2)$ & $7.9(4.9)$ & $5.3(3.5)$ & 0.74 \\
CD25 (IL-2 receptor) & $1.9(1.3)$ & $2.3(1.3)$ & $1.7(1.4)$ & 0.69 \\
HLA-DR & $1.4(1.7)$ & $4.5(2.6)$ & $22.2(23.5)$ & 0.009 \\
\hline
\end{tabular}

NK = natural killer; IL-2 = interleukin-2.

$\star$ From Ratjen. ${ }^{13}$

Group 1: immunocompromised patients with bacterial or fungal infection; group 2: immunocompromised patients without organisms in BAL fluid.

+ Wilcoxon test, group $2 v$ in controls.

children and responsible for considerable morbidity. ${ }^{1}$ The range of pathogens encountered is wide and includes bacterial, viral, fungal, and opportunistic organisms such as $P$ carinii. Most infections are treated successfully with broad spectrum antimicrobial therapy. ${ }^{14}$ For those patients not responding to empiric treatment, BAL offers the opportunity to sample material from the locus of infection. As demonstrated in this study, we were able to detect infectious organisms in a significant proportion of these children despite pretreatment with broad spectrum antibiotics. This is in agreement with previous studies in immunosuppressed children $^{210}$ and supports the usefulness of BAL in this population.

In addition, significant alterations were observed in differential cell counts of BAL fluid in immunocompromised children with pulmonary infiltrates. As in normal children, there was a predominantly neutrophil response to both bacterial and fungal infections, whereas lymphocytes were increased in the absence of bacterial or fungal pathogens. No concomitant changes could be observed in the differential cell counts of blood leucocytes at the time of BAL. Thus, even though these children were unable to evoke a systemic response to the pulmonary infection, the composition of the cells obtained from the focus of infection reflected the expected cellular response to the infectious agent.

Previously, little has been known about the differential cell counts of BAL fluid in children. De Blic et al have found no differences in differential cell counts between children with AIDS and primary or secondary immunodeficiencies. ${ }^{2}$ However, data for specific pathogens were not reported. In addition, most patients had $P$ carinii or cytomegalovirus infections, pathogens that were not encountered in this study. In adults, higher neutrophil counts have been reported in immunocompromised patients with bacterial pneumonia in comparison with those with no detectable pathogens. ${ }^{11}$ Moreover, limited evidence suggests that even patients undergoing bone marrow transplantation who lack leucocytes in peripheral blood can generate a local immune reaction in response to infectious agents. ${ }^{16} 17$ These results are in agreement with this study where we found marked cell proliferation on the bronchoalveolar surface despite systemic leukopenia. Although considerable variability was seen in BAL cytology in response to the pathogens, these data indicate that the differ- ential cell count on the bronchoalveolar surface may be of value in the differential diagnosis of infiltrates in immunocompromised hosts.

As we have previously demonstrated in children without lung disease CD8 cells are the predominant lymphocyte cell type encountered in BAL of normal subjects. ${ }^{15}$ This was not the case in children with negative BAL cultures in this study who had more CD4 cells in BAL fluid and therefore a CD4/CD8 ratio in excess of 1 . However, significant interindividual variability was observed largely due to a markedly higher value $(\mathrm{CD} 4 / \mathrm{CD} 8$ ratio $=7.8)$ in one child who underwent bone marrow transplantation and had engraftment on the day of lavage. Overall, the analysis of lymphocyte surface markers rendered little additional information to the differential diagnosis in the study population.

In summary, we have shown that BAL is useful in detecting pathogens in immunosuppressed children with pulmonary infiltrates despite pretreatment with broad spectrum antibiotics. In addition, significant differences were observed in BAL differential cell counts between children with bacterial or fungal infections and those with negative BAL cultures. Children with bacterial or fungal infection had higher neutrophil cell counts whereas lymphocytes were increased in children in whom no organisms were cultured from BAL fluid.

Accordingly, children with an increased percentage of neutrophils had a high probability of bacterial or fungal organisms in BAL fluid whereas a predominance of lymphocytes was associated with negative BAL cultures in most cases. These data suggest that the analysis of differential cell counts in BAL fluid can be of value in the differential diagnosis of pulmonary infiltrates in immunocompromised children. Further studies are warranted to assess the response of BAL cells to specific pathogens.

1 Williams DM, Remington JS. Pulmonary infection in the immunocompromised host. Am Rev Respir Dis 1976; 114: 259-64.

2 De Blic J, McKelvie P, Le Bourgois M, Blanche S, Benoist $M R$, Scheinmann $P$. Value of bronchoalveolar lavage in the management of severe acute pneumonia and interstitial management of severe acute pneumonia and interstitial
pneumonitis in the immunocompromised child. Thorax
$1987 ; 42: 759-65$.

3 Frankel LR, Smith DW, Lewiston NJ. Bronchoalveolar lavage for the diagnosis of pneumonia in the immunocomage for the diagnosis of pneumonia in the
promised child. Pediatrics 1988; 81: 785-8.

4 Stokes DC, Shenep J, Parham D, Bozeman PM, Marienchek W, Mackert PW. Role of flexible bronchoscopy in the diagnosis of pulmonary infiltrates in pediatric copy in the diagnosis of pulmonary infiltrates in
patients with cancer. $\mathcal{F}$ Pediatr $1989 ; 115: 561-7$. 
5 De Blic J, Blanche S, Danel C, Le Bourgeois M, Caniglia M. Bronchoalveolar lavage in HIV infected patients with
interstitial pneumonitis. Arch Dis Child 1989; 64: 1246-50.

6 Mattey JE, Brown Fitzpatrick S, Josephs SH, Fink RJ. Bronchoalveolar lavage for pneumocystis pneumonia in HIVchoalveolar lavage for pneumocystis pneumoni
infected children. Ann Allergy 1990; 64: 393-7.

7 Winthrop AL, Waddell T, Superina RA. The diagnosis of pneumonia in the immunocompromised child: use of bronchoalveolar lavage. $\mathcal{F}$ Pediatr Surg 1990; 25: 878-80.

8 Birrel JA, Adams JA, Saldana MA, et al. Role of bronchoscopy and bronchoalveolar lavage in the diagnosis of pediatric acquired immunodeficiency syndrome-related pulmonary disease. Pediatrics 1991; 87: 897-9.

9 Abadco DL, Amaro-Galvez R, Rao M, Steiner P. Experience with flexible bronchoscopy with bronchoalveolar lavage as a diagnostic tool in children with AIDS. Am 7 Dis Child 1992; 146: 1056-9.

10 McCubbin MM, Trigg ME, Hendricker CM, Wagener JS Bronchoscopy with bronchoalveolar lavage in the evaluation of pulmonary complications of bone marrow tion of pulmonary complications of bone marrow 43-7.

11 Sternberg RI, Baughman RP, Dohn MN, First MR.Utility of bronchoalveolar lavage in assessing pneumonia in immunosuppressed renal transplant recipients. $A m \mathcal{F} \mathrm{Med}$ 1993; 95: 358-64.

12 Ratien F, Bredendiek M, Brendel M, Meltzer S, Costabel U. Differential cytology of bronchoalveolar lavage fluid in normal children. Eur Respir $\mathcal{F} 1994$; 7: 1865-70

13 Ratjen F, Bredendiek M, Zheng L, Brendel M, Costabel U. Lymphocyte subsets in bronchoalveolar lavage fluid of children without bronchopulmonary disease. Am $\mathcal{F}$ Respir Crit Care Med 1995; 152: 174-8.

14 Pizzo PA. Management of fever in patients with cancer and treatment induced neutropenia. N Engl f Med 1993; 328 1323-32.

15 Costabel U, Bross KJ, Matthys H. The immunoperoxidase slide assay. A new method for the demonstration of surface antigens on bronchoalveolar lavage cells. Bull Eur Physiopathol Respir 1985; 21: 381-7.

16 Milburn HJ, DuBois RM, Prentice HG, Poulter LW. Pneumonitis in bone marrow transplant recipients results from a local immune response. Clin Exp Immunol 1990; 81: 232-7.

17 Leskinen R, Taskinen E, Volin L Tukainen P, Ruutu T Häyry $P$. Use of bronchoalveolar lavage cytology and determination of protein contents in pulmonary complications of bone marrow transplant recipients. Bone Marrow Transplant 1990; 5: 241-5. 